[Original article]

\title{
Total transferrin in cerebrospinal fluid is a novel biomarker for spontaneous intracranial hypotension
}

\author{
Junko Iijima $^{1) *}$, Kyoka Hoshi ${ }^{1) *}$, Hiromi Ito $^{1) *}$, Mayumi Kanno ${ }^{1)}$, Yuta Murakami ${ }^{2)}$, \\ Koichi Takahashi ${ }^{3)}$, Kana Matsumoto ${ }^{4}$, Yoshiki Yamaguchi $^{4)}$, Madoka Nakajima ${ }^{5}$, \\ Masakazu Miyajima ${ }^{5)}$, Hajime Arai ${ }^{5)}$, Mitsuyasu Kanai ${ }^{6)}$, Shinobu Kitazume ${ }^{7}$, \\ Takashi Honda ${ }^{8)}$ and Yasuhiro Hashimoto ${ }^{1)}$ \\ Departments of ${ }^{1)}$ Biochemistry, ${ }^{2)}$ Neurosurgery, ${ }^{7)}$ Preparing Section for the School of Health Sciences, and \\ ${ }^{8)}$ Human Life Sciences, Fukushima Medical University, 1 Hikarigaoka, Fukushima City, Fukushima \\ 960-1295, Japan, ${ }^{3)}$ Department of Neurosurgery, Sanno Hospital, 8-10-16 Akasaka, Minato-ku, Tokyo \\ 107-0052, Japan, ${ }^{4}$ Structural Glycobiology Team, Systems Glycobiology Research Group, RIKEN Global \\ Research Cluster, 2-1 Hirosawa, Wako City, Saitama 351-0198, Japan, ${ }^{5)}$ Department of Neurosurgery, \\ Juntendo University, 2-1-1 Hongo, Bunkyo-ku, Tokyo 113-8421, Japan, ${ }^{6)}$ Division of Neurology, Mihara \\ Memorial Hospital, Isezaki City, Gunma 372-0006, Japan
}

(Received August 31, 2020, accepted June 15, 2021)

\begin{abstract}
Spontaneous intracranial hypotension (SIH) is caused by cerebrospinal fluid (CSF) leakage. Patients with SIH experience postural headaches, nausea, etc., due to CSF hypovolemia. Imaging studies and clinical examinations, such as radioisotope (RI) scintigraphy, are useful for diagnosing SIH. However, 20-30\% of patients do not show typical morphology and clinical test results. We previously reported that CSF contains transferrin (Tf) isoforms: "brain-type" Tf derived from the choroid plexus and "serum-type" Tf derived from blood. We showed that both isoforms increased in the CSF of patients with SIH by Western blotting. In the present study, we demonstrate that conventional ELISA for quantifying total Tf is useful for diagnosing SIH more accurately than Western blotting. In addition, SIH with chronic subdural hematoma (CSDH) was also accurately diagnosed. Total Tf in the CSF can serve as a useful biomarker for diagnosing SIH with or without CSDH.
\end{abstract}

Key words : chronic subdural hematoma (CSDH), enzyme-linked immunosorbent assay (ELISA), spontaneous intracranial hypotension $(\mathrm{SIH})$, transferrin $(\mathrm{Tf})$

\section{Introduction}

Cerebrospinal fluid (CSF) physically cushions the brain and the spinal cord. Normally, CSF circulates and is constantly replenished. CSF is produced by the choroid plexus and secreted into the ventricles of the brain. The constant secretion of CSF contributes to vital functions such as providing nourishment, waste removal, and protection to the brain.

Spontaneous intracranial hypotension $(\mathrm{SIH})$ is caused by CSF leak, and the resulting CSF hypovo-

\footnotetext{
*These authors equally contributed to the present study. Corresponding author : Yasuhiro HashimotoＥmail : yasuc@fmu.ac.jp

Present affiliations and addresses :

Mayumi Kanno, Takashi Honda, Yasuhiro Hashimoto : Department of Forensic Medicine, Fukushima Medical University School of Medicine, 1 Hikarigaoka, Fukushima City, Fukushima 960-1295, Japan

Yoshiki Yamaguchi : Laboratory of Pharmaceutical Physical Chemistry, Tohoku Medical and Pharmaceutical University, 4-4-1 Komatsushima, Aoba-ku, Sendai City, Miyagi 981-8558, Japan (C)2021 The Fukushima Society of Medical Science. This article is licensed under a Creative Commons [Attribution-NonCommercial-ShareAlike 4.0 International] license.

https://creativecommons.org/licenses/by-nc-sa/4.0/
} 
lemia leads to orthostatic headaches. Clinical evaluation shows low intracranial pressure (ICP) and diffuse pachymeningeal enhancement on magnetic resonance imaging $(\mathrm{MRI})^{1-4)}$. Brain MRI and radioisotope (RI) cisternography can be used to identify SIH. Although these clinical tests are useful, 20$30 \%$ of patients with $\mathrm{SIH}$ do not show the typical manifestations ${ }^{3)}$. New tests and biomarkers are required for accurate diagnosis and timely intervention.

Transferrin ( $\mathrm{Tf}$ ) is an iron-binding protein secreted from the liver that carries iron to other organs via the blood stream ${ }^{5,6)}$. In previous studies, we identified two isoforms of Tf, with different glycosylation patterns, in the CSF. One isoform was designated as "serum-type" Tf, or Tf-2, which has glycans like those of serum transferrin, suggesting that the isoform is derived from blood. The other isoform, designated as "brain-type" Tf, or Tf-1, was detected in the CSF but not in serum ${ }^{7-9)}$. Braintype Tf was undetectable in the CSF of patients with hydranencephaly, lacking cerebral hemispheres, suggesting that brain-type $\mathrm{Tf}$ is of hemispheric origin ${ }^{10)}$. We found that both isoforms were increased in the CSF of patients with $\mathrm{SIH}^{11)}$, suggesting that Tf isoforms could be a new diagnostic marker for SIH.

Although each isoform was quantified by Western blotting, this method is time- and labor-consuming. In contrast, conventional ELISA is fast, sensitive, reproducible, and operationally high-throughput, but it does not distinguish Tf isoforms. This study aimed to demonstrate the usefulness of "total" $\mathrm{Tf}$ quantified by conventional ELISA in diagnosing SIH.

\section{Materials and methods}

\section{Patients}

We consecutively recruited patients suspected of SIH by lumbar puncture at the Sanno Hospital, as previously described ${ }^{11)}$. In brief, patients were diagnosed by clinical presentation, particularly orthostatic headache, MRI and/or computed tomography (CT), and RI scintigraphy. The diagnosis of SIH was based on the International Classification of Headache Disorders, 3rd edition (beta version) ${ }^{12)}$ and the diagnostic criteria reported by Schievinket $e t$ $a l .{ }^{13)}$ as follows: (1) Morphological evidence of CSF leakage such as pachymeningeal enhancement on cranial MRI (Fig. 1) and/or low CSF opening pressure $\left(\leq 60 \mathrm{~mm} \mathrm{H}_{2} \mathrm{O}\right)$. (2) No recent history of dural puncture. (3) Not attributable to another disorder. Non-SIH patients were defined as those suspected of SIH based on clinical presentation, but not fulfilling any of the above criteria. This study was approved by the Ethics Committees of Sanno Hospital (Approval No. 14-S-17 and 14-S-18) and Fukushima Medical University (Approval No. 2466), which are guided by local policy, national law, and the World Medical Association Declaration of Helsinki.

\section{CSF}

CSF samples from 150 patients were analyzed. Tf isoforms, rather than total Tf, were analyzed in 58 of 150 patients by Western blot in our previous study; these data were included in the present study, along with 92 new Western blot cases, all 150
A

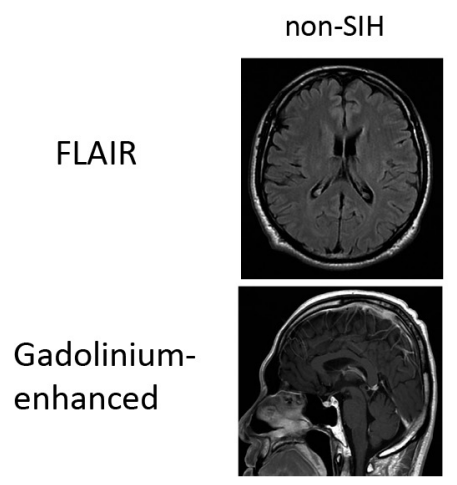

B

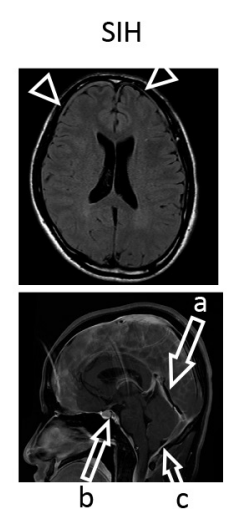

C

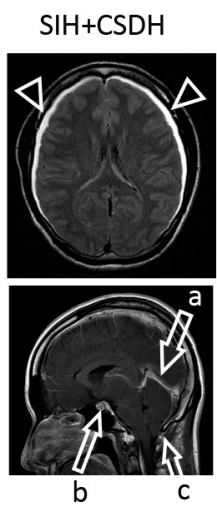

Fig 1. Cranial magnetic resonance imaging of patients without spontaneous intracranial hypotension (non-SIH), $\mathrm{SIH}$, and $\mathrm{SIH}$ with chronic subdural hematoma $(\mathrm{SIH}+\mathrm{CSDH})$.

MRI image of a non-SIH patient (A). Diffuse pachymeningeal gadolinium enhancement (arrowhead in the upper panel) and enlarged pituitary, cerebellar tonsil descent, and intracranial venous dilatation (arrows a, b, c respectively, in the lower panel) is shown with an SIH patient (B). In addition to these findings, a patient with $\mathrm{SIH}+\mathrm{CSDH}$ patient has subdural hematoma (arrowhead in the upper panel) (C). 
of which were also investigated with conventional ELISA. Patients were categorized into non-SIH, $\mathrm{SIH}$, or SIH with $\mathrm{CSDH}(\mathrm{SIH}+\mathrm{CSDH})$ groups. In the non-SIH group, there were 35 subjects (16 males and 19 females) with a mean age of $36.9 \pm$ 18.4 years (range, 9-72 years). SIH (SIH without $\mathrm{CSDH}$ ) was diagnosed in 57 cases (20 males and 37 females), mean age $39.4 \pm 9.0$ years (range, 26-64 years). $\mathrm{SIH}+\mathrm{CSDH}$ was diagnosed in 58 cases (36 males and 22 females), mean age $48.9 \pm 11.3$ years (range, 20-77 years). CSF samples were withdrawn after the measurement of ICP, and were centrifuged to remove cells and debris. What remained was stored in polypropylene tubes at $-80^{\circ} \mathrm{C}$ until analysis.

\section{Conventional ELISA}

Total concentration of $\mathrm{Tf}$ was measured in CSF as follows. A 96-well plate was coated with anti-Tf polyclonal antibody (Bethyl laboratories, $1: 1,000$ ) at $4^{\circ} \mathrm{C}$ overnight, and blocked with $0.5 \%$ bovine serum albumin (BSA) for $2 \mathrm{~h}$ at room temperature. Each CSF sample ( $1: 6,000-70,000$ dilution) was added and incubated for $2 \mathrm{~h}$ at $20^{\circ} \mathrm{C}$, and then incubated with a horseradish peroxidase (HRP)-conjugated goat antihuman $\mathrm{Tf}$ antibody (Bethyl laboratories, $1: 20,000)$ for $2 \mathrm{~h}$ at $20^{\circ} \mathrm{C}$. The signal was detected with a TMB Microwell Peroxidase Substrate System (KPL, 50-76-11) and quantified by Plate CHAMELEON (Hidex), with apo-Transferrin (Sigma, T4382) used as a standard.

\section{Western blotting}

We quantified $\mathrm{Tf}$ isoforms, brain-type and serum-type (formerly Tf- 1 and Tf- 2 , respectively) by immunoblotting as previously described ${ }^{11)}$. Briefly, each CSF sample was dissolved in sample buffer and boiled for $5 \mathrm{~min}$. The CSF sample was loaded onto 7.5\% SDS-polyacrylamide gels (SuperSep ${ }^{\mathrm{TM}}$ Ace; Wako Pure Chemical Industries, Osaka, Japan) and transferred to nitrocellulose membranes. After blocking the membrane with $3 \%$ skim milk in phosphate-buffered saline with Tween (PBST), and incubating sequentially with an anti-Tf antibody (Bethyl Laboratories) and an HRP-labeled anti-goat IgG (Jackson ImmunoResearch Laboratories, West Grove, PA), the protein was detected using a SuperSignal West Dura Chemiluminescence Substrate Kit (Pierce Biotechnology, Rockford, IL). Signal intensities were quantified by chromato-scanning with a CS Analyzer 2.0 (ATTO, Tokyo, Japan).

\section{Measurement of ICP and RI residual activity}

ICP and RI residual activity in the CSF was calculated as previously described ${ }^{11)}$.

\section{Statistical analyses}

Statistical analyses for non-SIH, SIH and SIH $+\mathrm{CSDH}$ were determined to be non-parametric by the Kruskal-Wallis test. We checked for correlations between total $\mathrm{Tf}$ and the two $\mathrm{Tf}$ isoforms or two clinical tests, and ICP and RI residual activity, using Spearman's rank correlation coefficient. Statistical analyses were performed using SPSS Statistics software (IBM Japan).

\section{Results}

\section{MRI of non-SIH, SIH, and SIH+CSDH patients}

Medical imaging is valuable for the diagnosis of CSF leakage. SIH has some characteristic imaging features ${ }^{14)}$. In Fig. 1, cranial MRI reveals that diffuse pachymeningeal gadolinium enhancement is observed in SIH (Fig. 1B, in the upper panel), and that typical signs of intracranial hypotension are also observable; i.e., intracranial venous dilatation, enlarged pituitary, and cerebellar tonsil descent (a, b, and c, respectively, in the lower panels of Fig. 1B and $\mathrm{C}$ ). In addition, subdural hematoma was observed in a patient with $\mathrm{SIH}+\mathrm{CSDH}$ (Fig. 1C, upper panel). Conversely, one non-SIH patient did not show morphological changes (Fig. 1A).

\section{Increase of Tf levels in the CSF of patients with SIH and $\mathrm{SIH}+\mathrm{CSDH}$.}

We analyzed $\mathrm{Tf}$ isoforms in the CSF by Western blotting: brain-type $\mathrm{Tf}(\mathrm{Tf}-1)$ derived from the choroid plexus and serum-type Tf (Tf-2) from blood. We previously reported that the levels of brain-type $\mathrm{Tf}$ and serum-type $\mathrm{Tf}$ were elevated in SIH. In patients with non-SIH, SIH, and $\mathrm{SIH}+\mathrm{CSDH}$, braintype $\mathrm{Tf}$ levels (mean \pm S.D.) were $7.7 \pm 3.5 \mathrm{ng} / \mu \mathrm{L}$, $14.7 \pm 7.1 \mathrm{ng} / \mu \mathrm{L}$, and $14.5 \pm 6.6 \mathrm{ng} / \mu \mathrm{L}$, respectively, whereas those of serum-type Tf were $18.9 \pm 6.0$ $\mathrm{ng} / \mu \mathrm{L}, 38.4 \pm 36.4 \mathrm{ng} / \mu \mathrm{L}$, and $38.0 \pm 22.6 \mathrm{ng} / \mu \mathrm{L}$, respectively. Subsequently, we investigated total Tf concentration in the CSF by conventional ELISA. As shown in Fig. 2, total Tf levels were higher in patients with $\mathrm{SIH}$ and $\mathrm{SIH}+\mathrm{CSDH}$ than in those with non-SIH. The total $\mathrm{Tf}$ levels were $16.4 \pm 7.2 \mathrm{ng} /$ $\mu \mathrm{L}$ in the non-SIH group, $42.1 \pm 33.6 \mathrm{ng} / \mu \mathrm{L}$ in the $\mathrm{SIH}$ group, and $42.0 \pm 28.4 \mathrm{ng} / \mu \mathrm{L}$ in the $\mathrm{SIH}+$ $\mathrm{CSDH}$ group (Fig. 2), indicating that conventional 
A

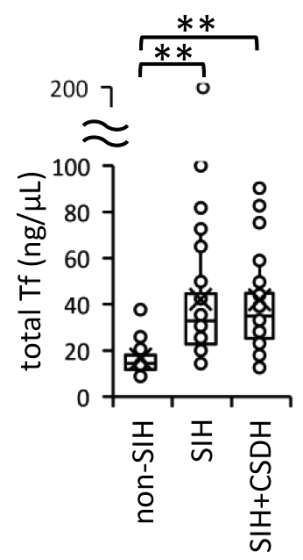

B

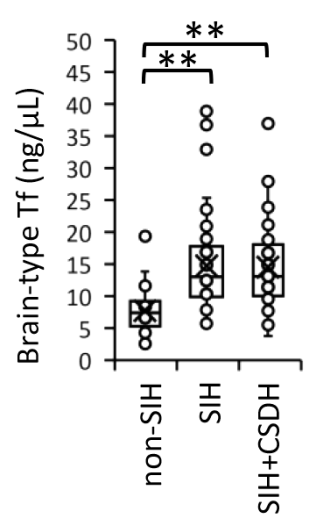

C

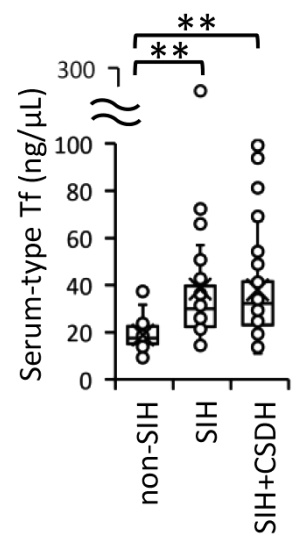

Fig 2. The levels of transferrin ( $\mathrm{Tf}$ ) in cerebrospinal fluid (CSF) are increased in patients with spontaneous intracranial hypotension $(\mathrm{SIH})$ and $\mathrm{SIH}$ with chronic subdural hematoma $(\mathrm{SIH}+\mathrm{CSDH})$.

Tf concentration was measured in the CSF of non-SIH $(n=35)$, SIH $(n=57)$ and SIH+CSDH $(n=58)$ patients. Total $\mathrm{Tf}$ is by conventional ELISA (A). Brain- and serum-type $\mathrm{Tf}$ is detected by Western Blotting (B, C). $* * p<0.01$

ELISA differentiates non-SIH from SIH and SIH+ $\mathrm{CSDH}$. No significant differences emerged in Tf concentrations of patients with SIH versus $\mathrm{SIH}+$ $\mathrm{CSDH}$ using the conventional ELISA or Western blotting.

Superiority of conventional ELISA over Western blotting as a diagnostic tool for $\mathrm{SIH}$ and $\mathrm{SIH}+\mathrm{CSDH}$.

Data obtained by conventional ELISA and Western blotting were statistically analyzed and compared for their diagnostic accuracy. We established the validity of conventional ELISA as a diagnostic tool for $\mathrm{SIH}$ and $\mathrm{SIH}+\mathrm{CSDH}$ by quantifying its sensitivity and specificity as a clinical test. In SIH, the sensitivity and specificity based on conventional ELISA data were $86.0 \%$ and $77.1 \%$, respectively. Western blotting data showed sensitivities and specificities in the range of $78.9-84.2 \%$ and $74.3-77.1 \%$, respectively (Table 1 ), indicating that conventional ELISA is more accurate than Western blotting. The diagnostic accuracy was also high for SIH+
CSDH. The combined data of all patients with SIH ( $\mathrm{SIH}$ and $\mathrm{SIH}+\mathrm{CSDH})$ indicates a sensitivity and specificity of $87.0 \%$ and $77.1 \%$ in conventional ELISA, versus $80.0-83.5 \%$ and $74.3-77.1 \%$ in Western blotting, respectively. Conventional ELISA showed high sensitivity and specificity in addition to being faster and having high throughput and reproducibility. These results show the superiority of conventional ELISA over Western blotting as a diagnostic tool for $\mathrm{SIH}$ and $\mathrm{SIH}+\mathrm{CSDH}$.

Correlation between the levels of total Tf and braintype Tf or serum-type Tf

The correlation coefficient of total Tf was examined with the Tf isoforms. Data of SIH and $\mathrm{SIH}+\mathrm{CSDH}$ were combined for the analysis (all $\mathrm{SIH}$ patients) (Fig. 3). In non-SIH, total Tf correlated well with Tf isoforms; $r=0.697$ for brain-type $\mathrm{Tf}, r=0.778$ for serum-type Tf, $r=0.776$ for braintype Tf + serum-type Tf (Fig. 3). The correlation coefficient of SIH and $\mathrm{SIH}+\mathrm{CSDH}$ (all patients with

Table 1. Sensitivity and specificity analysis.

\begin{tabular}{|c|c|c|c|c|c|c|c|}
\hline & & \multicolumn{2}{|c|}{$\mathrm{SIH}$} & \multicolumn{2}{|c|}{$\mathrm{SIH}+\mathrm{CSDH}$} & \multicolumn{2}{|c|}{ all SIH patients } \\
\hline & & $\mathrm{SN}(\%)$ & $\mathrm{SP}(\%)$ & $\mathrm{SN}(\%)$ & $\mathrm{SP}(\%)$ & $\mathrm{SN}(\%)$ & $\mathrm{SP}(\%)$ \\
\hline ELISA & Total Tf & 86.0 & 77.1 & 82.8 & 85.7 & 87.0 & 77.1 \\
\hline \multirow[t]{3}{*}{ WB } & Brain-type Tf & 78.9 & 77.1 & 81.0 & 80.0 & 82.6 & 74.3 \\
\hline & Serum-type Tf & 84.2 & 74.3 & 82.8 & 74.3 & 83.5 & 74.3 \\
\hline & Brain-type + Serum-type Tf & 80.7 & 77.1 & 79.3 & 77.1 & 80.0 & 77.1 \\
\hline
\end{tabular}

$\mathrm{SN}$ : sensitivity, SP : specificity, all SIH patients : Combined data of $\mathrm{SIH}$ and $\mathrm{SIH}+\mathrm{CSDH}$

$\mathrm{SIH}$ : spontaneous intracranial hypotension

$\mathrm{SIH}+\mathrm{CSDH}$ : spontaneous intracranial hypotension with chronic subdural hematoma 

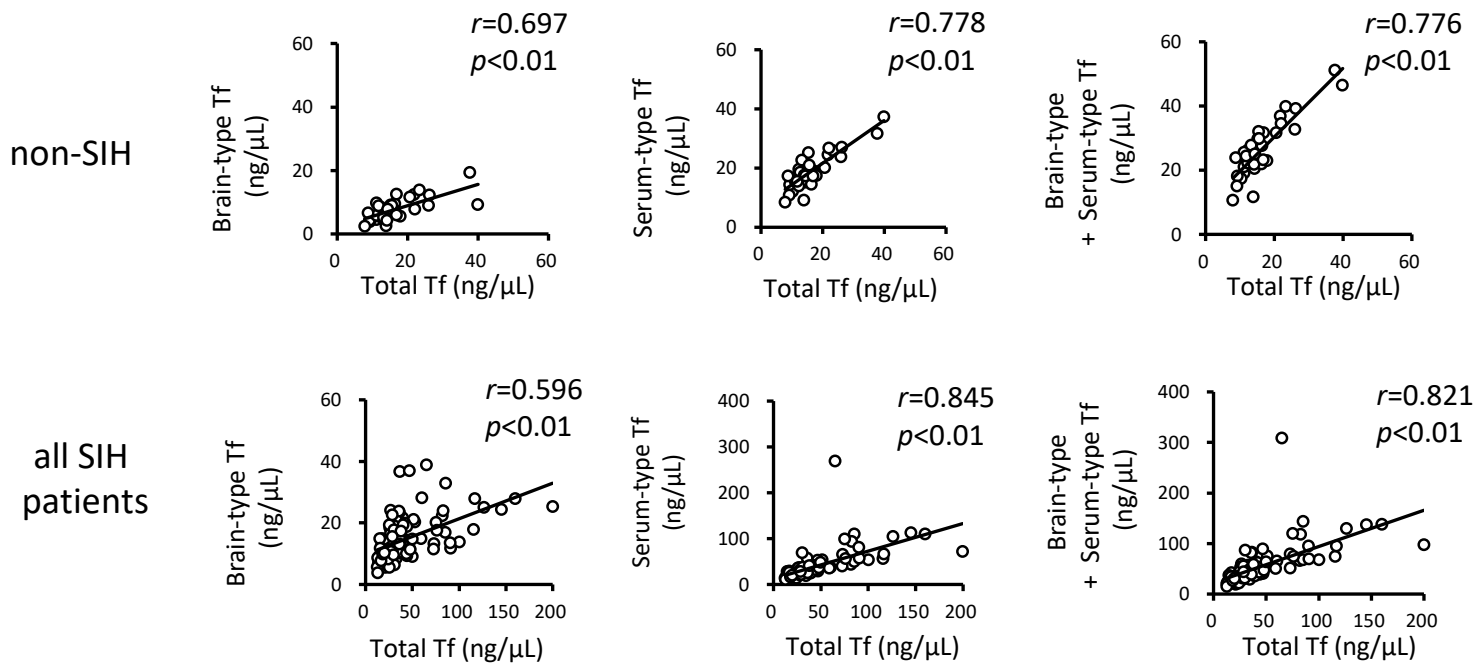

Fig 3. Correlation coefficient analysis on total transferrin (Tf) and $\mathrm{Tf}$ isoform levels.

Data obtained by conventional ELISA and Western blotting were subjected to correlation coefficient analysis. The cohort was divided into controls without spontaneous intracranial hypotension (non-SIH) and all SIH patients, which included SIH only and SIH with chronic subdural hematoma $(\mathrm{SIH}+\mathrm{CSDH}) . \quad$ Total $\mathrm{Tf}$ was measured by conventional ELISA. Brain and serum-type Tf were quantified by Western blotting.

$\mathrm{SIH})$ was $0.596-0.845$, and was similar to that of non-SIH.

\section{Correlation between total Tf and quantitative clinical tests for SIH.}

Measurements of ICP and RI residual activity have been established as diagnostic tools for differentiating SIH from non-SIH ${ }^{15,16)}$. These clinical tests were examined for their correlations with total Tf concentration in the CSF. Fig. 4 illustrates that a decrease in ICP is inversely correlated with an increase in total $\mathrm{Tf}$ in all SIH patient groups (combined data of SIH and $\mathrm{SIH}+\mathrm{CSDH})(r=-0.429, p<0.01)$ (Fig. 4, left panel). In addition, a decrease of RI residual activity is inversely correlated with an increase in Tf concentration in the CSF of these patients $(r=-0.414, p<0.01)$ (Fig. 4, right panel). In the SIH group, the cutoff of ICP was set at 9.7 $\mathrm{mm} \mathrm{H}_{2} \mathrm{O}$ and the cutoff of RI residual activity at $24 \mathrm{~h}$ after RI injection was set at $20 \%$. The cutoff of total $\mathrm{Tf}$ in all $\mathrm{SIH}$ groups was set at $17.96 \mathrm{ng} / \mu \mathrm{L}$. When the ICP cutoff is set at $9.7 \mathrm{~mm} \mathrm{H}_{2} \mathrm{O}$, all SIH patients is distinguished from non-SIH with $79.4 \%$ sensitivity and $65.1 \%$ specificity. When the cutoff of RI residual activity is set at $20 \%$, all SIH patients were differentiated from non-SIH with $64.7 \%$ sensitivity and $91.3 \%$ specificity. As shown in Table 1, total Tf shows $87.0 \%$ sensitivity and $77.1 \%$ specificity for the differentiation, indicating that total $\mathrm{Tf}$ is a supportive biomarker for diagnosing SIH including $\mathrm{SIH}+\mathrm{CSDH}$.

\section{Discussion}

The diagnosis of SIH is based on a combination of clinical features and imaging methods ${ }^{12,13}$. $\mathrm{SIH}$ is characterized by low CSF pressure and orthostatic headaches caused by CSF leakage but orthostatic headache is a non-specific complaint with variable manifestations. Medical imaging is valuable for the diagnosis of CSF leakage; e.g., cranial MRI reveals that SIH has characteristic anatomic features (Fig. $1)$.

In previous studies, we identified two isoforms of Tf, brain-type Tf and serum-type Tf, on Western blot (WB). Both isoforms were elevated in the CSF of patients with $\mathrm{SIH}^{11}$. This prompted us to investigate other laboratory methods to measure $\mathrm{Tf}$ in CSF, because WB is not suitable for routine clinical use due to time- and labor-consuming features. Our WB protocol takes 3 days, with a batch limit of 20 specimens. In addition, purified brain-type and serum-type standards are required for quantification. In contrast, conventional ELISA is widely available on commercial platforms, and the assay can be completed within a day. Clinical laboratory staff are usually familiar with ELISA. This facilitates its clinical utility for measuring $\mathrm{Tf}$ to diagnose SIH.

In Fig. 2, Western blotting and conventional ELISA showed that total Tf levels are elevated in $\mathrm{SIH}+\mathrm{CSDH}$ in addition to SIH, suggesting that total $\mathrm{Tf}$ could be a new diagnostic marker for SIH.

Although $\mathrm{Tf}$ isoforms are not distinguishable via conventional ELISA, this method is clinically 

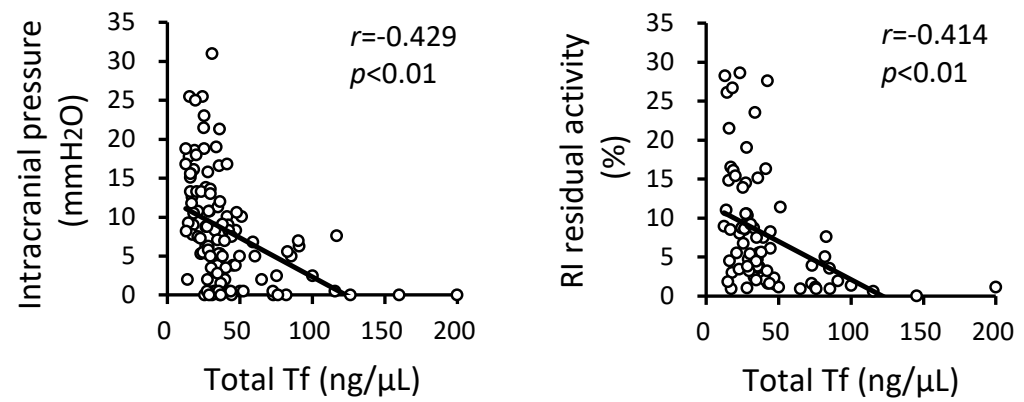

Fig 4. Correlation between CSF total transferrin (Tf) and clinical tests such as intracranial pressure (ICP) and radioisotope (RI) residual activity.

Decrease of ICP and RI residual activity were inversely correlated with increases of total Tf. Patients include those with spontaneous intracranial hypotension $(\mathrm{SIH})$ and $\mathrm{SIH}$ with chronic subdural hematoma $(\mathrm{SIH}+\mathrm{CSDH})$ (all SIH patients). Total Tf was measured by conventional ELISA.

valuable due to its speed, sensitivity, throughput, and reproducibility. A receiver operating characteristic (ROC) curve shows the relationship between clinical sensitivity and specificity; sensitivity indicates the ability to correctly generate a positive test result for persons who have the disease (true positive); specificity shows the ability to correctly generate a negative test result for persons who do not have the disease (true negative). From sensitivity and specificity analyses, conventional ELISA shows better accuracy than Western blotting for diagnosing $\mathrm{SIH}$ and $\mathrm{SIH}+\mathrm{CSDH}$ (Table 1). Next, we examine correlations between the levels of brain-type $\mathrm{Tf}$ and serum-type Tf, individually and combined (Fig. 3). The correlation coefficient of all patients with SIH is smaller than that of non-SIH. In particular, braintype Tf do not show high correlation $(r=0.523)$, suggesting that the levels of brain-type Tf are differentially controlled under CSF leakage. The correlation coefficient of total $\mathrm{Tf}$ is similar to that of serum-type Tf in non-SIH and all patients with $\mathrm{SIH}$, possibly because total $\mathrm{Tf}$ was mainly composed of serum-type Tf $(70 \sim 80 \%)$.

SIH is caused by CSF leakage from the subarachnoid space. The loss of CSF decreases ICP. CSF leakage can be estimated by RI cisternography. $\mathrm{RI}$ injected into the subarachnoid space rapidly decreases due to CSF leakage, resulting in low RI residual activity. Since RI cisternography and ICP measure have been established as diagnostic tools for differentiating SIH from non-SIH ${ }^{15,16)}$, we examine correlations between total $\mathrm{Tf}$ and the clinical tests for SIH (Fig. 4). A decrease in ICP or RI residual activity was inversely correlated with an increase of total $\mathrm{Tf}$ in all SIH patient groups. Furthermore, conventional ELISA for measuring total Tf yielded better ROC curves for the differential diagnosis than clinical tests. These results indicates the use of total Tf measured by conventional ELISA is a biomarker for diagnosing SIH including SIH+ CSDH. Conventional ELISA is an excellent system that can measure many samples simultaneously with few errors. It is expected that this system will contribute not only to the diagnosis of SIH, but also, to the assessment of other brain diseases in the future.

We showed that two isoforms of Tf increase in the CSF of patients with $\mathrm{SIH}$, and that total Tf can serve as a biomarker for $\mathrm{SIH}$ with and without CSDH. Beyond the immediate utility of transferrin as a biomarker for $\mathrm{SIH}$, our results indicate that iron-related conditions such as anemia and chronic infection should be further investigated in the context of patients with SIH and other neurological conditions.

\section{Acknowledgments}

This work was supported in part by the Japan Agency for Medical Research and Development (AMED) [grant numbers $16 \mathrm{hm} 0102042 \mathrm{~h} 0001$ and 17hm0102042h0002 and 18hm0102042h0003]; the Japan Science and Technology Agency [grant numbers AS221Z00232F, AS231Z01053, 241FT0255, ad 149]; the Ministry of Education, Culture, Sports, Science, and Technology of Japan [a Grant-in-Aid for Scientific Research on Innovative Areas, grant number 23110002 (Deciphering sugar chain-based signals regulating integrative neuronal functions), and a Grant-in-Aid for Scientific Research, grant number 23590367]; The General Insurance Association of Japan [a medical grant research on traffic accidents]; and the National Mutual Insurance Federation of Agricultural Cooperatives. 


\section{Conflict of interest disclosure}

The authors declare no conflict of interest relevant to this study or the findings outlined in this paper.

\section{Reference}

1. Mokri B, Posner JB. Spontaneous intracranial hypotension : the broadening clinical and imaging spectrum of CSF leaks. Neurology, 55 : 17711772, 2000.

2. Mokri B. Spontaneous intracranial hypotension. Curr Pain Headache Rep, 5 : 284-291, 2001.

3. Schievink WI. Spontaneous spinal cerebrospinal fluid leaks and intracranial hypotension. Jama, 295 : 2286-2296, 2006.

4. Mokri B. Spontaneous Intracranial Hypotension. Continuum (Minneap Minn), 21 : 1086-1108, 2015.

5. Crichton RR, Charloteaux-Wauters M. Iron transport and storage. Eur J Biochem, 164: 485-506, 1987.

6. Hentze MW, Muckenthaler MU, Galy B, Camaschella C. Two to tango : regulation of Mammalian iron metabolism. Cell, 142 : 24-38, 2010.

7. Futakawa S, Nara K, Miyajima M, et al. A unique $\mathrm{N}$-glycan on human transferrin in CSF : a possible biomarker for iNPH. Neurobiol Aging, 33 : 18071815, 2012.

8. Hoshi K, Matsumoto Y, Ito H, et al. A unique glycan-isoform of transferrin in cerebrospinal fluid : A potential diagnostic marker for neurological diseases. Biochim Biophys Acta Gen Subj, 1861 : 2473-2478, 2017.
9. Nagae M, Morita-Matsumoto K, Arai S, et al. Structural change of $\mathrm{N}$-glycan exposes hydrophobic surface of human transferrin. Glycobiology, 24: 693-702, 2014.

10. Murakami Y, Saito K, Ito H, Hashimoto Y. Transferrin isoforms in cerebrospinal fluid and their relation to neurological diseases. Proc Jpn Acad Ser B Phys Biol Sci, 95 : 198-210, 2019.

11. Murakami Y, Takahashi K, Hoshi K, et al. Spontaneous intracranial hypotension is diagnosed by a combination of lipocalin-type prostaglandin D synthase and brain-type transferrin in cerebrospinal fluid. Biochim Biophys Acta Gen Subj, 1862 : 1835-1842, 2018.

12. The International Classification of Headache Disorders, 3rd edition (beta version). Cephalalgia, 33 : 629-808, 2013.

13. Schievink WI, Dodick DW, Mokri B, Silberstein S, Bousser MG, Goadsby PJ. Diagnostic criteria for headache due to spontaneous intracranial hypotension : a perspective. Headache, 51 : 1442-1444, 2011.

14. Lin JP, Zhang SD, He FF, Liu MJ, Ma XX. The status of diagnosis and treatment to intracranial hypotension, including SIH. J Headache Pain, 18: 4, 2017.

15. Schievink WI, Maya MM, Louy C, Moser FG, Tourje J. Diagnostic criteria for spontaneous spinal CSF leaks and intracranial hypotension. AJNR Am J Neuroradiol, 29 : 853-856, 2008.

16. Monteith TS, Kralik SF, Dillon WP, Hawkins RA, Goadsby PJ. The utility of radioisotope cisternography in low CSF/volume syndromes compared to myelography. Cephalalgia, 36 : 1291-1295, 2016. 